\title{
A NEURAL NETWORK MODEL FOR PREDICTING COTTON YIELDS
}

\author{
Jun Zhang ${ }^{1}$, Yiming Wang ${ }^{2, *}$, Jinping $\mathrm{Li}^{1}$, Ping Yang ${ }^{1}$ \\ ${ }^{1}$ College of Information, Beijing Union University, 100101, Beijing, China \\ ${ }^{2}$ College of Information and Electrical Engineering, China Agricultural University, 100083, \\ Beijing, China \\ * Corresponding author, Address: P.O. Box 63, College of Information and Electrical \\ Engineering, China Agricultural University, 17 Tsinghua East Road, Beijing, 100083, P. R. \\ China, Tel: +86-10-62737591, Email: ym_wang@263.net
}

\begin{abstract}
Predicting a realistic target yield is one of the critical problems in precision farming. An artificial neural network was employed to model the nonlinear relationship between cotton yield and the factors influencing yield. Using sixyear field data obtained from LuoYang Dry Land Research Center, the neural network model was developed and trained, and the RMSE for test data was $3.70 \%$. The results indicate that the neural network model is a superior methodology for accurately setting cotton yields.
\end{abstract}

Keywords: artificial neural network, precision farming, cotton yield

\section{INTRODUCTION}

Factors affecting crop yield are so complex that traditional statistics cannot give accurate results. The artificial neural network can find non-linear relationships by observing a large number of input and output examples to develop a formula that can be used for predictions (Elizondo et al., 1994). Liu Jing et al. developed a neural network model for setting target corn yields and the influencing factors included soil properties, weather and management conditions (Liu et al., 2001). The ANN model resulted in higher $r^{2}$ and lower RMSE than the regression models (Kaul et al., 2005). In this research, a feed-forward BP ANN was employed to model the relationship between lint cotton yield and the factors influencing yield.

Zhang, J., Wang, Y., Li, J. and Yang, P., 2008, in IFIP International Federation for Information Processing, Volume 259; Computer and Computing Technologies in Agriculture, Vol. 2; Daoliang Li; (Boston: Springer), pp. 1321-1322. 


\section{MATERIALS AND METHODS}

Historical data from the six-year continuous field experiment (1999-2004) conducted in LuoYang Dry Land Research Center, Chinese Academy of Agricultural Sciences (CAAS) were used to build up the ANN model. Eleven input factors included management factors, soil properties, and weather factors. Eighteen records of eighteen subplots per year and six years of records provided the total set of 108 examples for the ANN model. Trial and error was used to select the parameter values that would give the most accurate predictions. The final network structure was 11-15-1 with the learning rate of 0.05 and the momentum coefficient of 0.6 . Initial weights and thresholds were generated randomly, and 11000 epochs were used in training. After 11000 epochs, the network was over-trained.

\section{RESULTS AND DISCUSSION}

The RMSE for the test examples was $3.70 \%$. Predicted yields were significantly related to the observed yields with the correlation coefficient reaching 0.9239 . The calculated yield shows the greatest sensitivity to the rainfall from July to August, the next greatest sensitivity to nitrogen fertilizer, and followed by rainfall from September to October. Growing season GDD and accumulated sunshine hours ranked fourth and fifth in influencing yield. The remaining factors showed less influence on the calculated yield.

\section{CONCLUSION}

The ANN model has high prediction accuracy and predicted yield trends versus the input factors are realistic. The ANN model has demonstrated here a promising potential in ecology. Further work is planned to test and retrain the ANN for a new field of larger size and with a variety of soil types.

\section{REFERENCES}

Elizondo D.A., McClendon R.W., Hoogenboom G. Neural network models for predicting flowering and physiological maturity of soybean. Transactions of the American Society of Agricultural Engineers, 1994, 37: 981-988

Kaul M., Hill R.L., Walthall C. Artificial neural networks for corn and soybean yield prediction. Agricultural Systems, 2005, 85: 1-18

Liu J., Tian L. A neural network for setting target corn yields. Transactions of the American Society of Agricultural Engineers, 2001, 44: 705-713 